\title{
Comportamiento reproductivo y mutilaciones en el cangrejo de las rocas Grapsus grapsus (Linnaeus, 1758) (Crustacea, Decapoda)
}

\section{Reproductive behaviour and mutilations in sally lightfoot Grapsus grapsus (Linnaeus, 1758) (Crustacea, Decapoda)}

\author{
Leonardo Romero*
}

Presentado: $20 / 11 / 2003$

Aceptado: $\quad 28 / 12 / 2003$

\begin{abstract}
Resumen
El presente trabajo analiza información de las mudas y observaciones del comportamiento reproductivo de G. grapsus, relacionando las frecuencias de mutilaciones y el comportamiento caníbal observado en las cópulas. Las colectas de mudas y observaciones sobre las cópulas se llevaron a cabo entre diciembre de 1990 y diciembre de 2001, en 200 m de una orilla rocosa al sur de la Bahía de Ancón, Lima, Perú. G. grapsus vive en grupos de número variable y asociados a grietas donde los individuos buscan refugio. El comportamiento de cortejo observado fue el de "búsqueda-intercepción" he incluyó diferencias como la defensa de territorios no temporales, largas persecusiones e intercepción por varios machos. Un total de 312 cópulas fueron observadas siendo más frecuentes durante el verano. Un total de once intentos de cópula produjeron la muerte de los individuos, siete fueron machos. En todo el periodo de estudio se colectaron un total de 8421 mudas de las cuales 418 presentaban mutilaciones o heridas en el cefalotórax o abdomen. No se observan diferencias significativas entre las tallas de mutilados de ambos sexos. El $73 \%$ de las mudas mutiladas presentan una sola mutilación, el $20 \%$ presentan dos mutilaciones y el $6 \%$ tres; se encontraron mudas hasta con siete apéndices mutilados. Las frecuencias de mutilaciones son significativamente mayores en machos. Las mutilaciones de las quelas y la segunda pata caminadora son más frecuentes en machos, mientras que en las hembras las mutilaciones de la cuarta y tercera pata caminadora son más frecuentes. Se propone que las mutilaciones se producen durante los intentos de cópula, principalmente por diferencias entre el tamaño de los individuos.
\end{abstract}

Palabras clave: Grapsus grapsus, comportamiento reproductivo, canibalismo, mudas, mutilaciones.

\section{Abstract}

We have analysed molt frequency and reproductive behaviour of $G$ grapsus in relation to mutilation frequency and cannibalism observed during mating. Observations and surveys were carried out between December 1990 and December 2001, on a 200 m stretch rocky shore in the south of Ancon Bay, Lima, Peru. Population of G. grapsus was organized in groups of variable numbers around crevices. The courtships behaviour of $G$ grapsus was based on the "search-interception" process including the defence of semi-permanent territory, along mate-tracking behaviour and interception by several males. A total of 312 copulation events were observed with the highest in summer. Eleven matings ended in death, seven victims of those being males. In this study 8421 molts were collected, 418 had mutilations or injuries in the cephalothorax or abdomen. There was no significant difference in the sizes of mutilated individuals between the two sexes. $73 \%$ of mutilated molts had one mutilation (injury), $20 \%$ two mutilation and $6 \%$ three mutilations and up to seven mutilations were recorded. Frequency of mutilation was significantly higher in males than in females. Mutilated appendices were different between sexes. Chela and second legs were more frequently mutilated in males and the fourth and third legs in females. We propose that mutilations observed were produced during copulation, manly due to size sizes.

Keywords: Grapsus grapsus, reproductive behavior, cannibalism, molt, mutilations.

\footnotetext{
* Departamento de Zoología, Facultad de Ciencias Biológicas, Universidad Nacional Mayor de San Marcos, Apartado 1898, Lima 1, Perú.

E-mail Leonardo Romero: Iromeroc@unmsm.edu.pe
} 


\section{Introducción}

Grapsus grapsus (Linnaeus, 1758) es una especie poco estudiada a pesar de su amplia distribución geográfica que va desde Baja California en EE.UU. hasta Talcahuano en Chile, incluyendo las Islas Galápagos y Juan Fernández. G. grapsus es una especie semiterrestre, común en la costa central del Perú; carnívora que se alimenta de cirrípedos, mitílidos, cangrejos porcellánidos y otros invertebrados; ocasionalmente también es carroñero. A pesar de ser una especie tan conspicua en las comunidades de orilla rocosa, se desconoce su ecología poblacional y la función que cumplen en estas comunidades.

Desde el punto de vista evolutivo las especies de la familia Grapsidae son muy interesantes ya que ocupan una gran diversidad de hábitats que van desde los ambientes intermareales hasta los bosques tropicales (Abele et al., 1986). Esta capacidad esta asociada al complejo comportamiento social que presentan, caracterizado por una gran variedad de señales y demostraciones ritualizadas (Seiple \& Salmon, 1982).

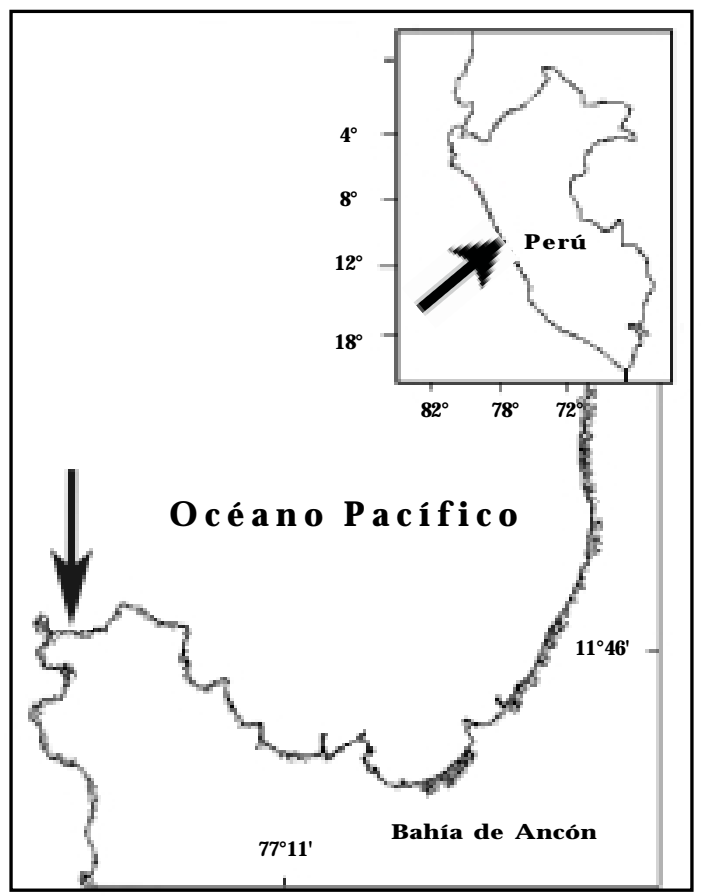

Figura 1. Mapa de la Bahía de Ancon. Flecha muestra la ubicacion de la área de estudio Ensenada del León
El comportamiento social y reproductivo se asocia en muchas especies al canibalismo es decir a la depredación de un individuo conespecífico. Este canibalismo en los crustáceos decápodos se relaciona a la autotomía, que es la capacidad voluntaria de perder un apéndice. Tanto el canibalismo como la autotomía en los artrópodos tiene especial importancia en el balance energético de la especie, constituyéndose en estrategias para optimizar el fitness (Chapman et al., 1999; Wasson et al., 2002). Desde hace mucho tiempo se ha conocido que las mutilaciones pueden producir retardo en el crecimiento de los individuos (Kuris \& Mager, 1975, Brock \& Smith, 1998). Además la intensidad de las mutilaciones pueden estar relacionadas a factores ambientales, geográficos y poblacionales (Smith \& Hines, 1991).

El presente trabajo analiza información recopilada de las mudas dejadas por los individuos de G. grapsus y de las observaciones de su comportamiento reproductivo, relacionándolos con las frecuencias de mutilaciones y el comportamiento caníbal observado en las cópulas.

\section{Material y métodos}

El estudio se llevo a cabo en una orilla rocosa denominada Ensenada del León al sur de la Bahía de Ancón ( $77^{\circ} 11^{\prime}$ W, 11 $46^{\circ}$ ' S) en Lima, Perú (Fig. 1). El área de estudio fue de 200 metros de orilla rocosa granítica, compuesta de macizos rocosos, peñones y una pequeña playa pedregosa. La comunidad predominante es la de mitílidos, los que forman franjas de ancho variable. Esta comunidad cambia en el tiempo alternando la dominancia de los mitílidos con algas. Aspectos de la zonación y fisonomía de la comunidad han sido descritos por Paredes (1974).

El periodo de estudio comprendió desde diciembre de 1990 a diciembre de 2001 . Se realizaron un total de 133 meses (484 días) de colectas y observaciones en el área de estudio. En el periodo de enero 1993 a marzo de 1994 (15 meses) no se realizaron colectas ni observaciones. 
El trabajo consistió en la colecta de los exoesqueletos o mudas que G. grapsus dejaba sobre las rocas, para ésto se realizaba una exhaustiva revisión de los 200 metros de ori1la. Las mudas eran medidas en el sitio con un calibrador Vernier $(0,05 \mathrm{~mm}$ de precisión). Las medidas tomadas fueron el largo del cefalotórax, el alto de la quela, el ancho mayor del abdomen, así como información del sexo, ubicación en el área de estudio, apéndices mutilados, heridas, etc. Los apéndices que aún no mostraban el tamaño normal, en el presente trabajo fueron considerados como apéndices mutilados. Luego de esto las mudas eran destrozadas para evitar volver a contarlas. Se estimó que una muda puede durar a la intemperie en promedio unos 20 días.

En el periodo de estudio además se realizaron observaciones ad livitum sobre el comportamiento de G. grapsus en particular sobre las cópulas. Estas observaciones tuvieron una duración que varió de 2 a 5 horas cada día y fueron realizadas entre las 11 y 18 horas. El principal motivo para limitar la duración de las observaciones fue la intensidad del oleaje.

Datos de temperatura de los archivos Pentad, fueron usados para obtener los promedios mensuales de temperatura superficial del área del Callao. Estos archivos son del programa TOGA y proporcionados por la NOAA (http://www.aoml.noaa.gov/phod/ epac/epac.html).

\section{Resultados y discusión}

\section{Comportamiento social y cortejo}

G. grapsus vive en grupos con número variable de individuos, que pueden llegar a tener más de 60 de diferentes tallas, siempre mayores de $3 \mathrm{~cm}$. Los grupos están asociados a grietas donde buscan refugio sobre todo cuando el oleaje es intenso. En el área de estudio se observaron cinco refugios claramente delimitados y permanentes durante el periodo de observaciones.

La mayoría de los individuos abandonan el refugio durante la marea baja para alimentarse. Durante la marea alta los individuos per- manecen sobre las rocas quietos y por lo general juntos con otros individuos de tamaños similares. Algunos individuos, machos o hembras realizaban caminatas que sobrepasaban los límites del área de estudio, sin embargo se observó que estos regresaban a sus respectivos refugios.

Kramer en G. grapsus de las Islas Galápagos observó el modelo de cortejo de «búsqueda - intercepción» (en Christy, 1987), en la cual los machos mantienen áreas temporales de cortejo y cópula, e interceptan a las hembras, las cuales pueden o no aceptar la cópula. Nosotros observamos además otras variaciones de este comportamiento:

1) Los individuos defendían los territorios que le servían de refugio; estos lugares podríamos considerarlos el recurso ofrecido a las hembras, esto se observó durante todo el periodo de estudio. En los refugios fue frecuente observar machos y hembras realizar demostraciones de agresividad. Estas demostraciones de agresividad podían ser realizadas por machos y hembras y consisten en movimientos verticales de las quelas con las puntas hacia abajo (Fig. 2). También se observó una demostración poco frecuente que sólo mostraron los machos, consistía en enfrentar al otro individuo (macho o hembra) y levantar las quelas y hacerlas girar en sentidos opuestos. Las demostraciones siempre se convertían en ataques a los individuos transeúntes que no eran del refugio.

2) También observamos cinco persecuciones, las que se caracterizaron por ser descontroladas, de tal manera que en una oportunidad un individuo que perseguía colisionó con el observador. En una oportunidad estimamos que la persecución fue por más de $50 \mathrm{~m}$. En los casos observados estas persecuciones terminaron en cópula.

3) En tres oportunidades observamos a un grupo de machos ( $4-5$ individuos) acorralar a la hembra y realizar cópulas consecutivas uno tras otro. 


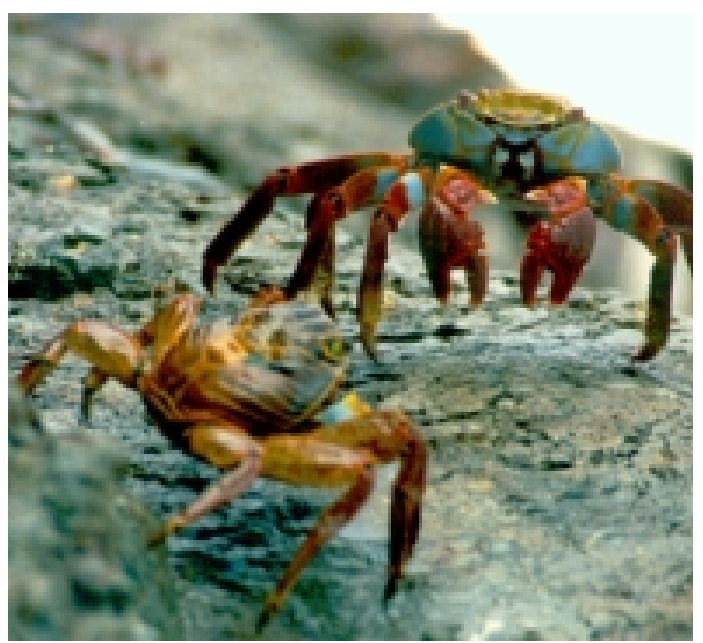

Figura 2. Demostración de agresividad de un macho de Grapsus grapsus. Bahía de Ancón.

Sin embargo la mayoría de veces el comportamiento de cortejo observado previo a la cópula fue un lento acercamiento, de la hembra y el macho, una demostración de agresividad del macho (Fig. 2), por último el macho colocaba sus quelas sobre las de la hembra y si no había respuesta negativa empezaba la cópula. En estos casos no existían territorios conspicuos, podían ocurrir en las áreas de comida (parte inferior del medio-litoral, durante la marea baja), descanso (zona supra-litoral) o muy cerca del refugio.

\section{Cópula y canibalismo}

La cópula empieza con la colocación del macho bajo la hembra (Fig. 3). La posición final es muy característica, la hembra mantiene sus quelas con las puntas hacia adentro, en forma horizontal y a la altura de la boca. El macho, ya bajo la hembra, coloca sus quelas con las puntas hacia arriba y a la altura de los ojos de la hembra, de manera que los meri sujeten las quelas de la hembra; las patas caminadoras del macho se entrelazan con las de la hembra. Las cópulas tienen una duración de 3 a 3,5 minutos, después de lo cual por lo general los individuos se separan y alejan.

Solamente seis veces se observaron cópulas múltiples entre los mismos individuos, estas se podían realizar exactamente en el mismo lugar o tener cortos desplazamientos.

Observamos canibalismo en 23 cópulas (7,4\% de las observaciones), once de estas agresiones causaron la muerte de los individuos, siete de ellos fueron machos. En las otras doce cópulas restantes los individuos atacados realizaban la autotomía de un apéndice para escapar y de esta manera no ser muerto por el individuo agresor.

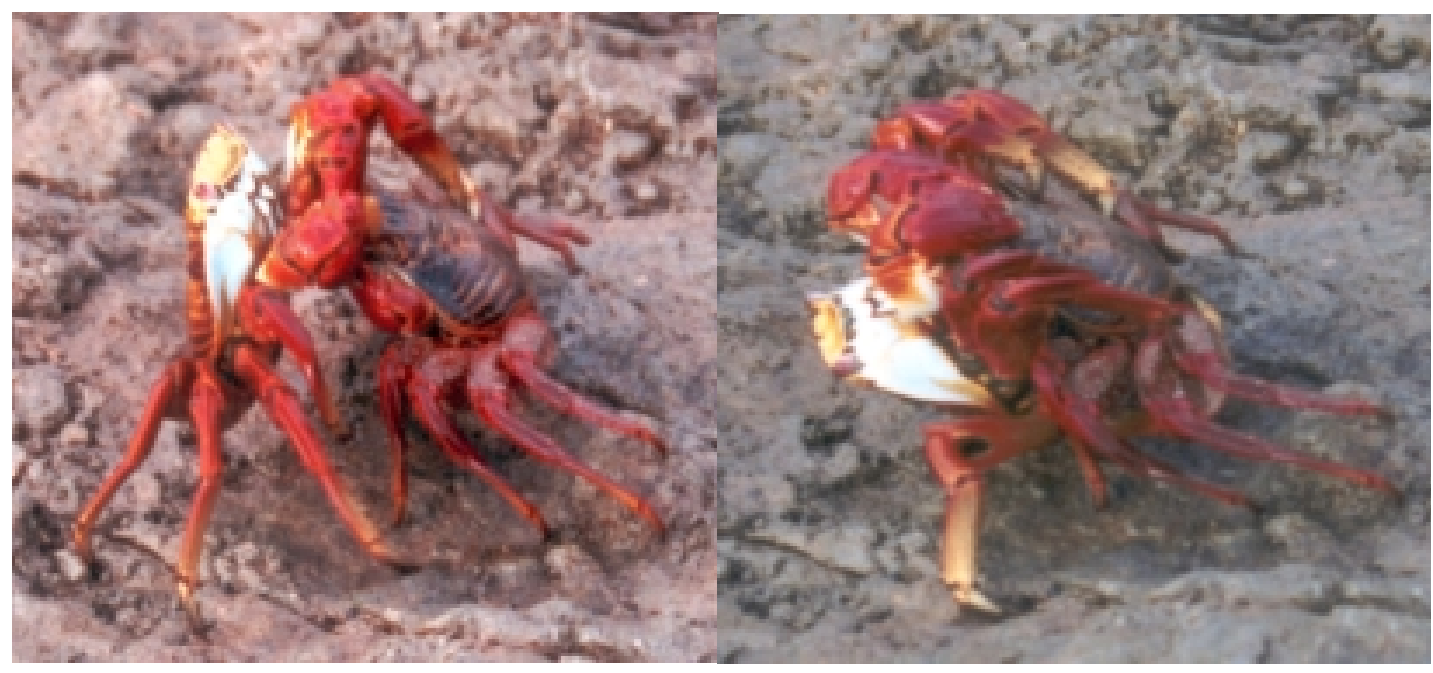

Figura 3. Cópula en Grapsus grapsus. Izquierda: el macho coloca sus quelas sobre la frente de la hembra y la toca con el segundo par caminador, la hembra tiene sus quelas delante de la boca. Derecha: macho se coloca bajo la hembra, usa las quelas para cubrir los ojos de la hembra, entrelaza sus patas con las de la hembra. 
Se observó que en todos los casos los individuos agresores eran más grandes que los agredidos, por lo general los machos intentaban iniciar la cópula aún siendo desproporcionadamente más pequeños y la agresión se realizaba en las primeras fases de la cópula. Observamos que es importante que el macho cubra los ojos de la hembra y sujete con los meri las quelas de la hembra, ya que esto permite bloquear su agresividad mientras que se acomoda debajo de ella. Si esto no ocurre la hembra se libera fácilmente y coge al macho. Los que fueron muertos eran cogidos por los flancos, lo que les producía inmovilidad y muerte casi instantánea, el agresor siempre abría el cefalotórax y se comía las gónadas luego una o tres patas.

En el caso que el agresor hubiere sido el macho, observamos que el menor tamaño de la hembra impedía que el macho logre desplazarse totalmente bajo ella y finalizar la cópula, a esto seguía la agresión del macho.

Estas observaciones nos indican que debe existir simetría entre los ejemplares que realizan la cópula. Aspectos morfológicos relacionados con el apareamiento serían muy importantes en el suceso de canibalismo.

Por otro lado estas agresiones tendría un efecto directo en la competencia intraespecífica en-
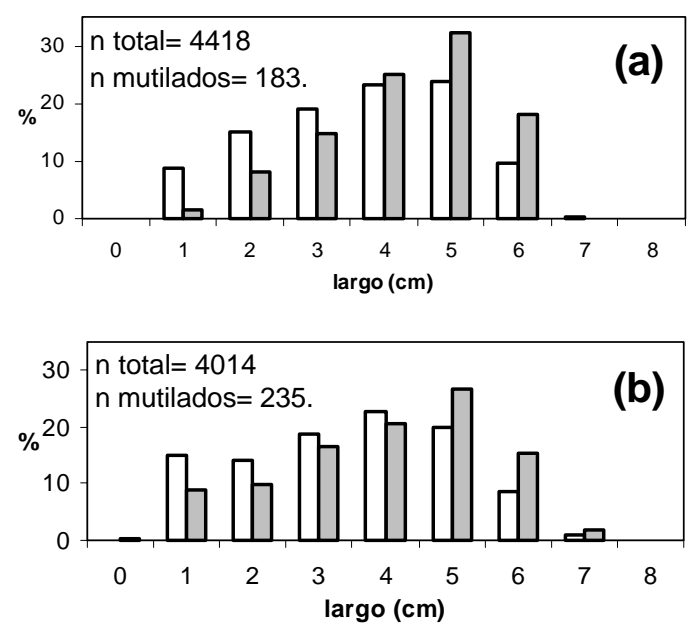

Figura 4. Distribucion de tallas de Grapsus grapsus. Barras blancas: total de mudas, barras grises: mutilados.(a) Hembras, (b) machos. tre grupos de diferentes edades. También hay que resaltar que el canibalismo observado no se ejecuta sobre un individuo del mismo sexo, por lo que la agresión no disminuiría la competencia sexual, y sólo podríamos relacionarlo con la disminución de la competencia intraespecífica entre grupos de edades diferentes.

\section{Proporción de sexos}

En todo el periodo de estudio se colectaron un total de 8421 mudas (4010 machos y 4411 hembras). De estas mudas 418 presentaban un apéndice mutilado o herida en el cefalotórax o abdomen (234 machos y 183 hembras). Para probar si existían diferencias significativas entre las proporciones sexuales se agruparon las mudas por años y se contrastaron los porcentajes de hembras y machos para cada año. El análisis muestra que existen diferencias significativas entre las frecuencias de mudas de machos y hembras (Kolmogorov-Smirnov, $\mathrm{D}=0,64 ; \mathrm{p}<$ $0,05)$; sin embargo no se encuentran diferencias significativas entre los sexos de los ejemplares mutilados (Kolgomorov-Smirnov, $\mathrm{D}=$ $0,18 ; \mathrm{p}=0,99)$.

Esto significa que en promedio para el periodo de observaciones se da un 5\% anual de mutilaciones y que no existen diferencias entre las incidencias de las mutilaciones en ambos sexos.

\section{Tallas}

Las tallas de G. grapsus fueron de 0,45 a 7,98 y 0,40 a $7,46 \mathrm{~cm}$ para los machos y hembras respectivamente. Mientras que los que presentaban algún tipo de mutilación estuvieron en los intervalos de 0,45 a 7,06 y 1,11 a $6,47 \mathrm{~cm}$ para los machos y hembras respectivamente (Fig. 4).

Agrupando las tallas por intervalos de un centímetro y obteniendo la frecuencias de los mutilados y no mutilados encontramos que existen diferencias significativas entre las dos distribuciones de frecuencias (Kolgomorov Smirnov $\mathrm{D}=0,67 ; \mathrm{p}<0,05$ para hembras y $\mathrm{D}=$ 0,$75 ; \mathrm{p}<0,01$ para machos) lo cual indica que 


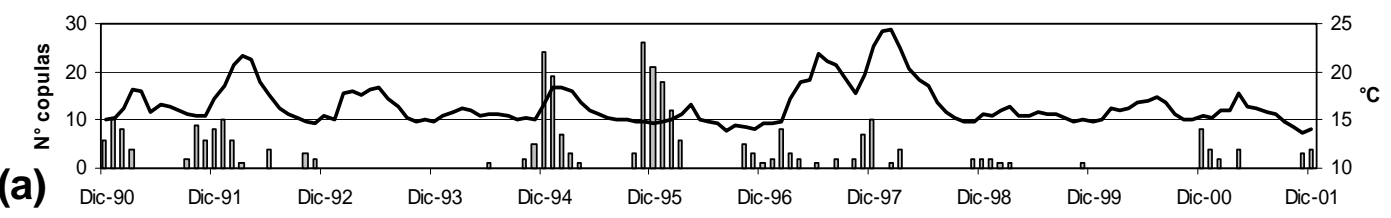

$\square$ Total de copulas $\longrightarrow$ Tcal

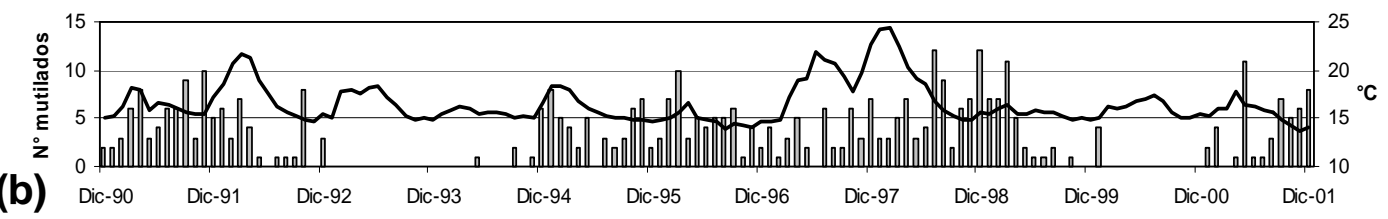

$\square$ Totalmut $\longrightarrow$ Tcal

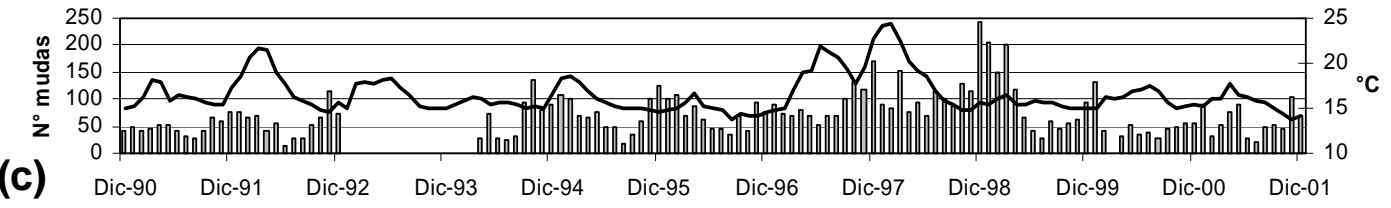

$\square$ Totalmudas $\longrightarrow$ Tcal

Figura 5. Series de tiempo de observaciones de frecuencias mensuales de cópulas, en barras (a), mutilados (b), y mudas colectadas (c) de Grapsus grapsus, en la Ensenada del León, Bahía de Ancón, entre diciembre 1990 y diciembre 2001. En línea sólida se muestra el promedio mensual de temperatura obtenida de los archivos Pentad de la NOAA.

las tallas de los mutilados tienden a ser mayores que el resto de la muestra analizadas. No se observar diferencias significativas entre los sexos de mutilados.

\section{Estacionalidad}

La información del número de mudas, mutilados y cópulas fue agrupada mensualmente obteniendo 133 meses.

Un total de 312 cópulas fueron observadas en el periodo de estudio, el máximo número de cópulas que se registró en un mes fue de 26. Las cópulas fueron observadas con mayor frecuencia durante el verano (Fig. 5a).

Se pudieron observar unos pocos casos (6) de cópulas en los meses de invierno y que se dieron en el periodo de El Niño 91 - 92 (4 casos), El Niño 97 - 98 (un caso) y uno en el año 1994.

Se observaron un total de 578 apéndices mutilados en 418 individuos en todo el periodo de estudio. El promedio mensual de mudas con mutilaciones fue de 5,3\% con un intervalo de $0-21 \%$. El promedio anual fue de $4,5 \%$ con un intervalo de $(2-6 \%)$.

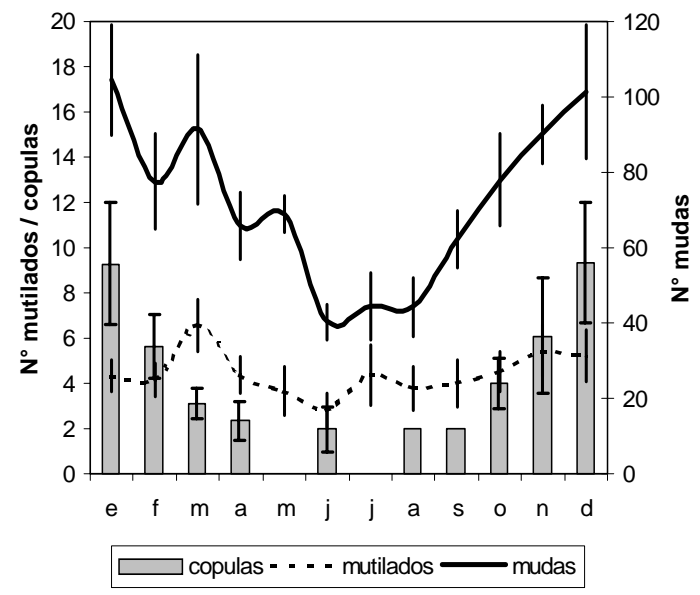

Figura 6. Promedios mensuales para el periodo de estudio, de diciembre 1990 a diciembre 2001, de cópulas, mutilados y mudas de Grapsus grapsus, en Bahía de Ancón. Barras verticales indican el error estandar. 
Tabla 1. Coeficientes de Correlación de Spearman $\left(r_{s}\right)$ comparando los valores promedios mensuales de Mudas, Mutilados, Cópulas para Grapsus grapsus, en la Bahía de An-

\begin{tabular}{lllll}
\hline cón. & & Mudas & Mutilados & Cópulas \\
\hline Mudas & $\mathrm{r}_{\mathrm{S}}$ & 1 & $0,673^{*}$ & $0,890^{* *}$ \\
& $p$ & - & 0,017 & 0,001 \\
& $\mathrm{~N}$ & 12 & 12 & 10 \\
Mutilados & $\mathrm{r}_{\mathrm{S}}$ & $0,673^{*}$ & 1 & 0,620 \\
& $p$ & 0,017 & - & 0,056 \\
& $\mathrm{~N}$ & 12 & 12 & 10 \\
Cópulas & $\mathrm{r}_{\mathrm{S}}$ & $0,890^{* *}$ & 0,620 & 1 \\
& $p$ & 0,001 & 0,056 & - \\
& $\mathrm{N}$ & 10 & 10 & 10 \\
\hline
\end{tabular}

${ }^{* *}$ Correlación significante en $p<0,01$ (2-colas). ${ }^{*}$ Correlación significante en $p<0,05$ (2-colas).

La serie de mutilaciones presenta mayor variabilidad temporal, no observándose ciclos estacionales. Mientras que la serie de mudas presentan ciclos estacionales que se incrementan en los meses de verano (Fig. 5 b, c).

Las series de los promedios de temperatura no se correlacionan significativamente con las frecuencias de mudas, mutilados y cópulas.

Para el análisis de la estacionalidad se agruparon los datos en meses multianuales, obteniéndose el promedio de mudas, mutilados y cópulas para cada mes. Se puede observar una clara tendencia estacional en las variables de mudas y cópulas (Fig.6). Esta agrupación proporciona valores de correlación altas y significativas entre las mudas y mutila-

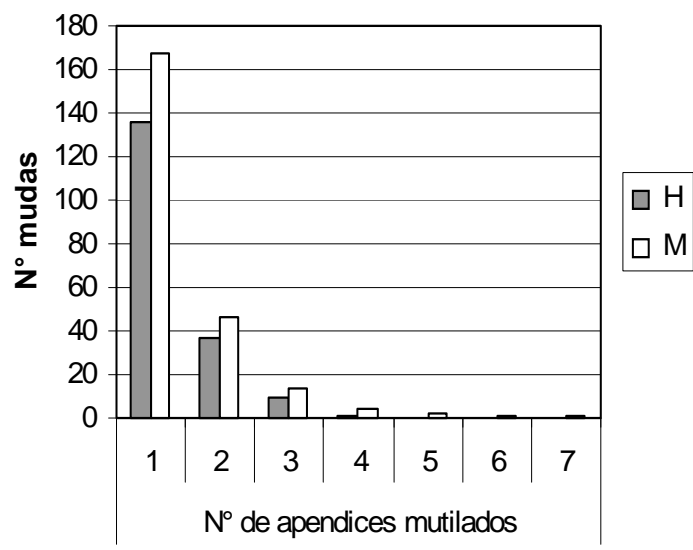

Figura 7. Frecuencias del número de apéncides mutilados de Grapsus grapsus, en Bahía de Ancón. dos y entre las mudas y cópulas pero no entre mutilados y cópulas (Tabla 1).

La estacionalidad observada en la frecuencia de mudas puede relacionarse con los periodos reproductivos, afirmación reforzada en la correlación positiva y significativa que existe con las observaciones de frecuencias de cópulas. Se ha observado para otras especies de Grápsidos que durante estos periodos suelen incrementar la frecuencia de mudas y pueden tener hasta cuatro puestas durante el periodo (López y Rodriguez, 1998).

La mayor frecuencia de cópulas entre primavera y verano estaría en relación a los ciclos de anuales de mareas y en especial con la ampliación del área de alimentación mas que con los valores temperatura.

Por otro lado la baja y no significativa correlación de las cópulas con las mutilaciones podría explicarse porque las observaciones de las mutilaciones son posteriores al suceso en sí. La mayor frecuencia de mutilaciones en marzo y julio indicarían los intervalos de desfase relacionados con la duración del periodo de intermuda.

\section{Apéndices mutilados}

La autotomía es un mecanismo frecuentemente utilizado como medio de escape de un

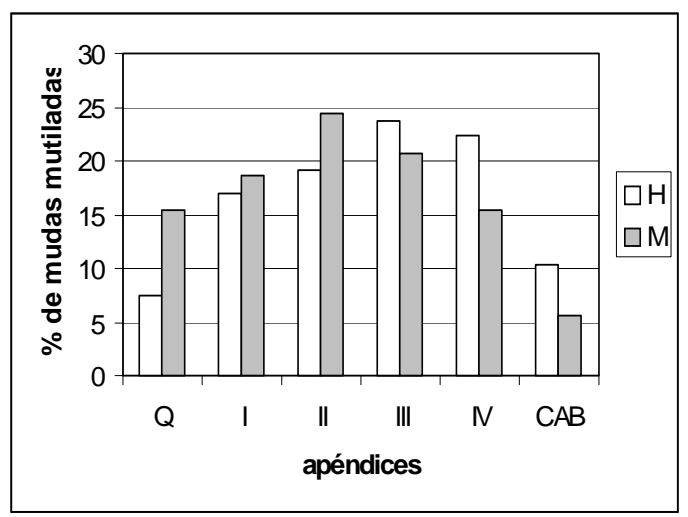

Figura 8. Porcentaje de frecuencia de mudas mutiladas por apéndice en Grapsus grapsus. $\mathrm{H}=$ hembras, $\mathrm{M}=$ machos. $\mathrm{Q}=$ quelas. I, II, III, IV= primera, segunda, tercera y cuarta pata caminadora. 
depredador, sin embargo en el área de estudio, G. grapsus es una presa ocasional para algunos depredadores como Larus belcheri, Heliaster helianthus y el hombre. Estos depredadores ocasionales son muy exitosos, por lo que podemos asumir que la presencia de mutilados en la población estudiada se debe casi exclusivamente a los encuentros agresivos intraespecíficos.

El $73 \%$ de las 418 mudas presentan una sola mutilación, el $20 \%$ dos y el $6 \%$ tres mutilaciones.Han podido encontrarse mudas hasta con 7 apéndices mutilados (Fig. 7). No se observa diferencias significativas entre la incidencia en el número de apéndices mutilados y el sexo $\left(\chi^{2}=4,63 ; p=0,59\right)$. Si consideramos las mutilaciones de los apéndices, las heridas en el cefalotórax y en el abdomen observamos que solamente han sido registrados 68 combinaciones de las 144 posibles.

Si consideramos la frecuencia de mutilación en cada uno de los apéndices, no observamos diferencias significativas entre el lado derecho e izquierdo ya sea en machos o hembras $(\mathrm{F}=0,08 ; p<0,78)$. Sin embargo las frecuencias de mutilaciones son mayores significativamente en machos $(\mathrm{F}=9,16 ; p<$ 0,008) (Fig. 8).

También podemos observar diferencias entre las frecuencias del apéndice mutilado con respecto del sexo $\left(\chi^{2}=17,9 ; p<0,01\right)$. La mutilaciones de las quelas son más importantes en machos, mientras que las mutilaciones en la cuarta caminadora son más importantes en las hembras. Esta diferencia estaría relacionada con la posición de los individuos en la cópula. Los machos serían los más expuestos a las agresiones, sus apéndices delanteros, las quelas y el segundo par caminador serían los más propensos a ser mutilados. En cambio las hembras sufren menos mutilaciones y son los apéndices posteriores, el cuarto y tercer caminador los afectados.

\section{Agradecimientos}

El autor agradece los comentarios y sugerencias de Mutsunori Tokeshi. El presente trabajo fue financiado parcialmente por el CSIUNMSM con el FEDU.

\section{Literatura Citada}

Able L. G.; P. J. Campanella and M. Salmon. 1986. Natural history and social organization of the semiterrestrial grapsid crab Pachigrapsus transversus. J. Exp. Mar. Biol. Ecol. 104: 153 170.

Brock R. E. and L. D. Smith. 1998. Recovery of Claw Size and Function Following Autotomy in Cancer productus (Decapoda: Brachyura). Biol. Bull. 194: 53-62.

Chapman J. W.; T. Williams, A. Escribano, P. Caballero, R. D. Cave, and D. Goulson. 1999. Fitness consequences of cannibalism in the fall armyworm, Spodoptera frugiperda. Behav. Ecol. 10: 298-303.

Chisty J. H. 1987. Competitive mating, mate choice and mating associations of brachiuran crabs. Bulletin of Marine Science, 4 (2): 177 - 191.

DeRivera C. E. and S. L. Vehrencamp. 2001. Male versus female mate searching in fiddler crabs: a comparative analysis. Behav. Ecol. 12: 182-191.

Kuris A. M. and M. Mager. 1975. Effect of limb regeneration on size increase at molt of the shore crabs Hemigrapsus oregoniensis and Pachygrapsus crassipes. J. Exp. Zool. 193: $353-360$.

López L. S. and E. M. Rodríguez. 1998. Somatic growth in juveniles of the estuarine crab Chasmagnathus granulata Dana 1851 (Brachyura, Grapsidae), under laboratory conditions. Invest. Mar. 26: 127-135,

Paredes C. 1974. Modelo de Zonación en la orilla rocosa del Departamento de Lima. Rev peru Biol.. 1(2):168 - 191

Seiple W. and M. Salmon. 1982. Comparative social behavior of two grapsid crabs, Sesarma reticulatum and S. cinereum. J. Exp. Mar. Biol. Ecol. 62: 1 - 24.

Smith L. D. and A. H. Hines. 1991. Autotomy in blue crabs (Callinectes sapidus Rathbun) populations: Geografic, temporal and ontogenetic variation. Biol. Bull. 180: 416 - 431

Wasson K.; B. E. Lyon, and M. Knope. 2002. Hair-trigger autotomy in porcelain crabs is a highly effective escape strategy. Behav. Ecol. 13: 481-486. 